\title{
Noninvasive Transient Elastography identifies initial stage liver fibrosis \& cirrhosis in Hepatitis B patients
}

\author{
Muhammad Naveed Anwar, Humaira Achakzai, Fahim Ullah, Rizwan Amin Kundi, Zeeshan Ayaz, \\ Asif Imran
}

\section{Submitted}

April 23, 2020

Accepted

June 05,2020

\section{Author Information}

From: Department of Medicine, Rehman Medical Institute, Peshawar, Khyber Pakhtunkhwa, Pakistan

Dr. Muhammad Naveed Anwar

Consultant Gastroenterologist (Corresponding Author)

Email:

naveed.anwar@rmi.edu.pk

Dr. Humaira Achakzai Consultant Physician

Dr. Fahim Ullah

Consultant Endocrinologist

Dr. Rizwan Amin Kundi Consultant Physician

Dr. Zeeshan Ayaz

House Officer

Dr. Asif Imran

Registrar Gastroenterology

Citation: Anwar MN, Achakzai H, Ullah F, Kund RA,Ayaz Z, Imran A. Noninvasive Transient Elastography identifies initial stage liver fibrosis \& cirhosis in Hepatitis B patients. J Rehman Med Inst. 2020 AprJun;6(2):3-7.

\begin{abstract}
Background: Among the many non-invasive techniques performed on patients with Hepatitis B Virus (HBV) related fibrosis, the most appropriate results have been obtained using Transient Elastography (FibroScan) to measure liver stiffness. The accurate diagnosis of fibrosis related to $\mathrm{HBV}$ is essential for prognostic and therapeutic decisions and offset the limitations of liver biopsy.
\end{abstract}

Objective: To evaluate Transient Elastography (FibroScan) for detecting and staging hepatic fibrosis by comparing it with hepatic histopathology in patients with Hepatitis B at a tertiary care hospital of Peshawar.

Materials \& Methods: A descriptive study was done in March-April 2020 at Rehman Medical Institute, Peshawar, based on retrospective data from May 2016 to March 2020 on $145 \mathrm{HBV}$ positive patients with various stages of hepatic fibrosis who had undergone noninvasive FibroScan during their first visit. Routine demographic, clinical and laboratory parameters were analyzed to predict the existence or absence of advanced fibrosis. At the end of the evaluation, the samples were categorized as F0 (No fibrosis), F1 (Initial fibrosis without septa), F2 (Fibrosis with septa), F3 (Advanced fibrosis) and F4 (Severe fibrosis with cirrhosis, C1) Descriptive data analysis was done by SPSS 17.0.

Results: Out of 145 included patients of chronic hepatitis B, 110 were males and 35 were females. At initial stages the existing laboratory tools diagnosed chronic HBV-infected patients correctly, with minor fibrosis and cirrhosis. Independent indicators of liver fibrosis included platelet count, age, AST, ALT values and albumin; $80 \%$ of the patients were not affected by the disease, which means F0 was $80 \%$. Yet some patients had fibrosis at different stages F1, F2, F3, F4 and C1. The FibroScan assessment revealed that out of 145 patients, only $11 \%$ patients were at stage $\mathrm{F} 1$ whereas the diagnostic value for $\mathrm{F} 2, \mathrm{~F} 3, \mathrm{~F} 4$, and $\mathrm{C} 1$ are $\leq 3 \%$ with $\mathrm{F} 2=3 \%, \mathrm{~F} 3=1 \%, \mathrm{~F} 4=2 \%$, and $\mathrm{C} 1=2 \%$.

Conclusion: FibroScan is an effective method to detect $\mathrm{HBV}$-related fibrosis and cirrhosis; it provided accurate distinction between various stages of fibrosis in Hepatitis B patients.

Keywords: Cirrhosis, Liver; Hepatitis B; Liver Fibrosis; Carcinoma, Hepatocellular.

The authors declared no conflict of interest. All authors contributed substantially to the planning of research data collection, data analysis, and write-up of the article, and agreed to be accountable for all aspects of the work.

\section{INTRODUCTION}

Chronic HBV is a primary reason of liver diseases and deaths worldwide. Not all 250 million individuals who are chronically infected with $\mathrm{HBV}$ are likely to undergo serious harm (require therapy) or develop complications of severe liver diseases including hepatocellular carcinoma (HCC) and decompensated cirrhosis without intervention. ${ }^{1} \mathrm{HBV}$ is primarily liver-centered, where a cycle of hepatocyte damage and tissue repair ensues due to contact of viral proteins with the immune system of the body. In this repair, repeated accumulation of extracellular matrix leads to growth of severe liver fibrosis over time. The increase in advanced fibrosis may be fast, slow, or intermittent depending on the state of disease and the level of active liver injury and inflammation. Formal evaluation of liver fibrosis is important for diagnosis of the disease and for assessing urgency and response to treatment. The main indicator of the result is the intensity of liver disease. Cirrhosis results in lower survival rate and enhanced occurrence of HCC. ${ }^{2}$ Patients without cirrhosis have a survival rate of $97 \%$ to $63 \%$, whereas cirrhosis patients have survival rates of $55 \%$ to $25 \% .^{3}$

Based on the complicated natural history of HBV infection, patients need professional evaluation to correctly stage the disease, understand viral serology, biochemistry, and start monitoring or required treatment. ${ }^{4}$ Non-invasive markers are helpful supplements for demonstrating the treatment results without repetitive liver biopsies. In recent years, non-invasive tests have been introduced to reduce the need for liver biopsy and enhance clinical practice. ${ }^{6}$ Identification of liver fibrosis by Transient Elastography (TE) FibroScan in HBV patients is an important factor in disease management and prognosis. ${ }^{1} \mathrm{TE}$ is one of the best non-invasive techniques and it diagnoses HBV related fibrosis by measuring liver stiffness. $^{5}$ It can be done effectively in the outpatient clinic or at the bedside with instant results. Limitations to the method include only $5 \%$ cases of failure which were mostly recorded in patients with excessive thoracic fat. 
FibroScan is preferred for the early evaluation of liver fibrosis in formerly untreated chronic HBV patients.

FibroScan has been accepted worldwide as the tool to analyze and evaluate mild and severe fibrosis (cirrhosis) in HBV/HCV patients, HIV-HCV co-infections and reoccurrence of hepatitis $\mathrm{C}$ following liver transplantation. FibroScan is the most appropriate method for diagnosis of early fibrosis and assessment of portal hypertension, which can also be predictive. Studies are needed using FibroScan to check two types of patients i.e. patients who are taking treatment and the other who are not. It also requires monitoring of the patients who are at risk of liver disease. Although FibroScan is a useful technique that measures the stiffness of the liver, its values must be interpreted on the basis of clinical, biological and morphological data.

\section{Chronic Hepatitis Grading \& Staging}

Both staging and grading are frequently used in chronic viral hepatitis. In general, the creation of scoring systems to be used in liver diseases has exceeded the demonstration of clinical utility. The grade of disease reflects the rapid progress towards the end stage of the disease. While the stage of disease measures the degree to which it has advanced in its natural history, leading to organ failure or death of the patient. The initial stages have lower level of fibrosis or cirrhosis, whereas the end-stage in majority of chronic liver diseases is cirrhosis with clinical decompensation. The grade of the disease is the degree of infection and hepatocellular injury that is supposed to result in fibrosis in chronic necro-inflammatory diseases (HBV, HCV \& HDV) and autoimmune hepatitis. The grade represents the intensity of existing liver disease with characteristics that alter according to the type of injury and its pattern. Ideally, grade and stage both must predict the diagnosis and direct therapeutic intervention, but in most of the chronic liver diseases the evidence is relatively minor. Moreover, treatment can modify the natural history in an unspecified manner. However, pathologists are often asked to allocate the stage as well as grade in the process of assessment of fibrosis to find out a patient's result. ${ }^{7}$

\section{Transient Elastography (FibroScan)}

There are special end-to-end non-invasive techniques that are often used to assess liver fibrosis. Transient Elastography (TE) is among the most commonly used global methods for quantifying liver fibrosis, even in chronic Hepatitis B and C. There is a transducer installed in the axis of a vibrator at the end of the probe which helps in directing $50 \mathrm{MHz}$ pressure waves on the specified liver tissue. As a result, painless vibrations spread a shear wave which represents the velocity of the wave being returned to the ultrasound. The shear wave is followed, monitored, and shown in kilopascals by means of a device, thus assessing the liver fibrosis. TE is a quick bedside test which is very simple to perform, with instant clinical follow-up. In fact, TE is the measurement of Liver Stiffness (LS) that depends on the level of fibrosis; it does not measure liver fibrosis directly.

Until now, this method has proven effective in evaluating fibrosis and diagnosing cirrhosis complexities like portal hypertension. ${ }^{8}$ Thus, a number of measurements are essential for correct assessment. Even so, the results of assessment prove to be challenging due to some limitations such as narrow intercostal space may increase false results, the patient have to hold the breath during the process for minimum errors, and the fasting conditions are essential as well. Another important factor is that one cannot perform FibroScan on patients of ascites. A meta-analysis correlated the FibroScan values with the METAVIR score on the patients of HBV. F2 has a value of 7.9 $\mathrm{kPa}$, and $\mathrm{F} 4$ was related to value $11.7 \mathrm{kPa}$ with a sensitivity of 0.859 and 0.929 respectively. ${ }^{9}$ However, some researches reflect that the outcomes in HBV might be influenced by aminotransferases flares confronted in affected patients. ${ }^{10}$ The European Association For the Study of the Liver (EASL) approved this method in management of viral hepatitis for its accuracy, non-invasiveness, and ability of differentiating between different stages of fibrosis. ${ }^{11}$

Limitations for transient elastography include steatosis of liver stiffness values and the conflicting impacts of inflammation activity. A machine, an operator training and costs associated with it are needed for TE. TE was initially verified for HCV instead of $\mathrm{HBV}$, with lesser cut-off values to identify fibrosis in HBV. While valid to diagnose cirrhosis, TE is facing decreased accuracy at lower fibrosis levels, same as in blood-based biomarkers, yet TE is still extremely useful addition to the clinical toolkit.

\section{AST/ALT ratio (AAR)}

There are various categories of non-invasive markers used to predict the intensity of HBV related fibrosis. Indirect markers use regular laboratory tests such as liver synthetic makers, transaminases or other readily available indicators which are relevant to the stage of liver diseases like red cell distribution width or platelet levels. In various etiologies of liver diseases, AAR is commonly used as a prognosticator of cirrhosis and the ratio of AST/ALT in ordinary subjects is around 0.8. This ratio may be effective as a non-invasive measure of cirrhosis and fibrosis in patients having chronic hepatitis B. ${ }^{12}$ However, Eminler and colleagues discovered that AAR performed less than other non-invasive blood algorithms when assessing the stage of fibrosis in 237 patients with HBV. Likewise, the potential of AAR to identify the advanced fibrosis (F2-F4) was limited in $319 \mathrm{HBV}$ patients in an US group. ${ }^{13,14}$

\section{MATERIALS \& METHODS}

\section{Patients}

This descriptive study was performed on retrospective data from May 2016 to March 2020 on patients examined at the Department of Radiology in Rehman Medical Institute, Peshawar. A total of 145 patients previously diagnosed with chronic Hepatitis B were registered in the study. Transient Elastography (FibroScan) test was done on these patients to study stages of fibrosis. The clinical follow-up provided physicians with information for the next diagnostic measures. Statistical and laboratory data of patients were gathered at the time of first outpatient visit or initial hospital admission. The rest of details were collected from the electronic medical 
records. Chronic HBV have been detected with the evaluation of hepatitis B surface antigen (HBsAg) for over six months. Patients who suffered from liver illness such as Hepatitis A, C, $\mathrm{E}$ infection, previous nucleoside treatment, $\mathrm{HCC}$, fatty liver disease, previous interferon therapy, ultrasonographic or clinical evidence of cirrhosis, and deficient liver tissue for the staging of fibrosis were excluded from the study.

\section{Laboratory Examination}

Every patient was subjected to complete clinical history along with laboratory and clinical examination which included liver function test such as serum albumin, bilirubin, and liver enzymes, INR, alpha-fetoprotein, HCV RNA via polymerase chain reaction, seromarkers for $\mathrm{HBV}$ (HBsAg) using ELISA method, prothrombin time and concentration, and complete blood count (CBC). ${ }^{15}$ Abdominal ultrasonography was performed on every patient to diagnose cirrhosis; tests for presence of ascites, splenic size and portal vein diameter were followed by FibroScan to measure liver stiffness.

\section{Grading and staging}

For Grading and staging of the disease, METAVIR scoring system was used.

\section{Liver Stiffness Measurement}

Various measurements were carried out on patients' right liver lobe, lying on the back with the full abduction of right arm. The transducer tip was coated with coupling gel and placed between the rib bones onto the skin at right liver lobe. With the help of ultrasound, the operator found a part of the liver of at least $6 \mathrm{~cm}$ thickness without huge vascular structures. After locating the measurement area, the operator pushed the button on probe to begin the procurement. The depth of measurement ranged from $25 \mathrm{~mm}$ to $65 \mathrm{~mm}$ under the skin surface. The software automatically rejected measurements which resulted in incorrect follow up of the vibration propagation or incorrect vibration shape. Every patient underwent up to 10 measurements and the success rate was estimated as a proportion of the amount of successful acquisitions relative to their sum, which showed the results in kilopascals $(\mathrm{kPa})$. The median value of successful measures was obtained to show liver stiffness. The liver stiffness measurement was considered reliable only if it had at least a 30\% success rate and at least five successful measurements. The entire test took less than five minutes. ${ }^{16}$

\section{Statistical and data analysis}

Statistical and Data analysis was conducted to evaluate relevant differences based on statistics using commercially software package SPSS version 17. ${ }^{15}$ Measurement units were described as mean \pm standard error of mean of data. The mean and standard deviation of FibroScan measurements was calculated for all fibrosis stages. The TE diagnostic value was calculated by histopathological fibrosis staging (METAVIR score) to diagnose all four stages of fibrosis. Here, F0 meant No Fibrosis, F1 meant Initial Fibrosis Without Septa, F2 meant Fibrosis With Septa, F3 meant Advanced Fibrosis, and F4 meant Severe Fibrosis With Cirrhosis (C1). The METAVIR scoring system was derived from individual factors for determining the level of fibrosis (scarring) in Hepatitis B patients.

\section{RESULTS}

The study included 145 patients of chronic hepatitis B, out of which 110 were males and 35 were females; ages of patients were from 17-70 years; 123 patients were examined on their initial visit whereas 22 patients were on follow-up visits.

Table 1 shows patients classified according to the METAVIR scoring system into F0 (116), F1 (16), F2 (05), F3 (02), F4 and $\mathrm{C} 1$ (03 each). The mean stiffness of liver was $4.5 \pm 0.2 \mathrm{kPa}$ for patients with F0 (range: $1.5-6.5$ ), $7.1 \pm 0.107 \mathrm{kPa}$ for patients with F1 (range: $6.5-7.5$ ), $8.2 \pm 0.30 \mathrm{kPa}$ for patients with F2 (range: 7.5-9.5), $11.5 \pm 0.5 \mathrm{kPa}$ for patients with F3 (range: 9.512.5 ), $13.8 \pm 0.61 \mathrm{kPa}$ for patients with $\mathrm{F} 4$ (range: $12.5-15.5$ ), and $20.8 \pm 0.45 \mathrm{kPa}$ for patients with $\mathrm{C} 1$ (range: $15.5 \&$ above).

Table 1: Results of FibroScan of patients based on the METAVIR scoring system ( $n=145)$.

\begin{tabular}{|c|c|c|c|c|c|c|}
\hline $\begin{array}{c}\text { Fibrosis } \\
\text { stages }\end{array}$ & Frequency & Percent & $\begin{array}{c}\text { Cumulative } \\
\text { Percent }\end{array}$ & $\begin{array}{c}\text { Mean } \\
\text { deviation }\end{array}$ & $\begin{array}{c}\text { Standard } \\
\text { Deviation }\end{array}$ & $\begin{array}{c}\text { Liver Stiffiness } \\
\text { (kPa) } \\
\text { (Mean } \pm \text { SD) }\end{array}$ \\
\hline F0 & 116 & 80.0 & 80.0 & 0.92 & 1.14 & $04.5 \pm 0.2$ \\
\hline F1 & 16 & 11.0 & 91.0 & 0.21 & 0.26 & $7.1 \pm 0.107$ \\
\hline F2 & 05 & 03.4 & 94.4 & 0.58 & 0.70 & $8.2 \pm 0.30$ \\
\hline F3 & 02 & 01.4 & 95.8 & 0.50 & 0.70 & $11.5 \pm 0.5$ \\
\hline F4 & 03 & 02.1 & 97.9 & 0.80 & 1.05 & $13.8 \pm 0.61$ \\
\hline C1 & 03 & 02.1 & 100.0 & 01.8 & 0.78 & $20.8 \pm 0.45$ \\
\hline Total & 145 & 100.0 & & & & \\
\hline
\end{tabular}

Table 2 shows the laboratory details. The mean values for AST and ALT were $40.3 \pm 7.53$, and $76.95 \pm 10.96$, respectively.

Table 2: Laboratory findings of included patients $(n=145)$.

\begin{tabular}{|l|c|}
\hline \multicolumn{1}{|c|}{ Test } & $\begin{array}{c}\text { Test Results } \\
(\text { Mean } \pm \text { SEM) }\end{array}$ \\
\hline AST (U/L) & $40.3 \pm 7.53$ \\
\hline ALT (U/L) & $76.95 \pm 10.96$ \\
\hline INR & $0.99 \pm 0.015$ \\
\hline Platelet Count $(\mathrm{cmm})$ & $24320.4 \pm 24056.6$ \\
\hline
\end{tabular}




\section{DISCUSSION}

The study showed that in patients with chronic Hepatitis B, Transient Elastography is an effective method for diagnosing fibrosis and its intensity. Moreover, the cut-off values of liver stiffness for various stages of liver fibrosis should be determined before carrying out the examination. The duration between the two tests should not be more than three months as it is mostly a valid time interval for the individuals with less severe fibrosis. Evaluators of the result should not be aware of the provided treatment. ${ }^{15}$

The meta-analysis of 27 studies in 2015 involved $4386 \mathrm{HBV}$ patients in which high diagnostic precision was demonstrated by TE to assess liver fibrosis. The summary of sensitivity was 0.81 , 0.82 , and 0.86 , the specificity values were $0.82,0.87$, and 0.87 , and the relevant AUROC values were 0.88, 0.91, 0.93 where fibrosis stages were $\mathrm{F} \geq 2, \mathrm{~F} \geq 3$, and $\mathrm{F}=4$, respectively. ${ }^{16}$ Significant fibrosis $(\geq \mathrm{F} 2)$ cut-off values ranged from $5.8-8.8$ $\mathrm{kPa}$, fibrosis with septa $(\geq \mathrm{F} 3)$ ranged from $7.0-13.5 \mathrm{kPa}$ and the cirrhosis (F4) ranged from $9.0-16.9 \mathrm{kPa}^{17-21} \mathrm{TE}$ has been shown to be effective for fibrosis evaluation in the latest research involving $263 \mathrm{HBV}$ patients having the ALT levels $<2 x$ Upper Limit of Normal (ULN), specifically ones diagnosed with significant fibrosis. The value of liver stiffness was observed to be substantially associated with liver fibrosis as well as necro-inflammatory activity, an option to explain TE measurments. ${ }^{22}$ It is proposed by few authors that cut-off values for TE should include ALT levels which change with the swelling and redness in HBV. In case of patients with negative $\mathrm{HBe} A g, \mathrm{TE}$ might be helpful to indicate the need for biopsy treatment. ${ }^{23}$ In HBV patients TE has been demonstrated as a prognosticator of liver-oriented results. Another analysis of 381 HBV patients (that were beginning the treatment) found growing accumulated incidence rates of HCC correlated with increased LS values in three graded groups including LS $<8$, LS $=8-13$, and LS $>13 \mathrm{kPa}$; LS was an autonomous HCC growth indicator instead of histological staging. ${ }^{24}$

For the patients having chronic HBV, hepatic fibrosis is a reversible scarring reaction which eventually results in cirrhosis and nodule formation. The correct assessment of fibrosis is necessary to guide management and predict diagnosis.
Histological assessment of liver biopsy stays the gold standard for quantification of fibrosis. Lately, there is a growing interest in the use of the non-invasive methods to provide more regular sampling and to prevent the occurrence of percutaneous biopsy. Several researchers have shown correlation between progression of fibrosis and activity grade to cirrhosis. The evaluation of fibrous scarring for the staging of liver disease has always been a key function of liver biopsy analysis. However, it is recently considered that fibrosis is an irreversible process, and the changes-with-time are relatively slow. Thus, there are few stages for most of the grading and staging systems and as a result they are extremely inadequate to describe changes in fibrosis.

In our current study, we have tried to determine the role of FibroScan in prognosis and staging of liver fibrosis in chronic HBV patients. In chronic HBV patients, numerous factors have been tested to evaluate liver fibrosis. Our research consisted of different clinical, laboratory, and demographic parameters to analyze liver fibrosis in patients of $\mathrm{HBeAg}$ positive chronic HBV with ALT and AST values. Our research included 145 patients that were serologically proved to have chronic HBV and were selected for the FibroScan to identify patients with fibrosis. There were a few participants with various intensities of liver fibrosis. Out of 145 patients, 116 had no fibrosis which means $80 \%$ of patients were not affected by fibrosis at all $(\mathrm{F} 0=80 \%)$. Only 16 patients were diagnosed with initial fibrosis without septa $(\mathrm{F} 1=11 \%)$ and 05 patients with few septa $(\mathrm{F} 2=3 \%)$. The remaining 8 patients had severe fibrosis out of which 2 patients had advanced fibrosis $(\mathrm{F} 3=1 \%), 3$ were at severe fibrosis stage with no cirrhosis $(\mathrm{F} 4=2 \%)$ and 3 had cirrhosis $(\mathrm{C} 1=2 \%)$. Therefore, total number of cirrhotic patients in our research was fairly small.

\section{CONCLUSION}

FibroScan (Transient Elastography) is a secure, rapid, and noninvasive method to obtain measurement of liver stiffness as it may be effective for staging and grading of liver fibrosis along with results. Liver stiffness measurement as a result of Transient Elastography has a good interconnection with METAVIR score for liver fibrosis.

\section{REFERENCES}

1. Parikh P, Ryan JD, Tsochatzis EA. Fibrosis assessment in patients with chronic hepatitis B virus (HBV) infection. Ann Translat Med. 2017;5(3):40-53.

2. Crossan C, Tsochatzis EA, Longworth L, Gurusamy K, Davidson B, RodríguezPerálvarez $\mathrm{M}$, et al. Cost-effectiveness of non-invasive methods for assessment and monitoring of liver fibrosis and cirrhosis in patients with chronic liver disease: systematic review and economic evaluation. Health Tech Assess. 2015;19(9):1-410.
3. de Lédinghen V, Vergniol J. Transient elastography (FibroScan). Gastroenterol Clin Biol. 2008 Sep;32(6 Suppl 1):58-67.

4. Papachrysos N, Hytiroglou P, Papalavrentios L, Sinakos E, Kouvelis I, Akriviadis E. Antiviral therapy leads to histological improvement of $\mathrm{HBeAg}$ negative chronic hepatitis B patients. Ann Gastroenterol. 2015;28(3). Accessed November 20, 2020. Available from: https://pubmed.ncbi.nlm.nih.gov/2612692 9/.

5. EASL-ALEH Clinical Practice Guidelines: Non-invasive tests for evaluation of liver disease severity and prognosis. J Hepatol. 2015;63(1):237-64.

6. Goodman ZD. Grading and staging systems for inflammation and fibrosis in chronic liver diseases. J Hepatol. 2007;47(4):598-607.

7. Jieanu $C F$, Ungureanu $B S$, Săndulescu DL, Gheonea IA, Tudoraşcu DR, Ciurea ME, et al. Quantification of liver fibrosis in chronic hepatitis $B$ virus infection. Journal of medicine and life. 2015;8(3):285-90. Accessed November 25, 2020. Available from: https://www.ncbi.nlm.nih.gov/pmc/articles /PMC4556907/\#R42 
8. Cosgrove D, Piscaglia F, Bamber J, Bojunga J, Correas J-M, Gilja OH, et al. EFSUMB Guidelines and Recommendations on the Clinical Use of Ultrasound Elastography.Part 2: Clinical Applications. Ultraschall in der Medizin European Journal of Ultrasound. 2013;34(03):238-253.

9. EASL Clinical Practice Guidelines: Management of hepatitis $\mathrm{C}$ virus infection. J Hepatol. 2011;55(2):245-64.

10. Li Y, Huang Y-S, Wang Z-Z, et al. Systematic review with meta-analysis: the diagnostic accuracy of transient elastography for the staging of liver fibrosis in patients with chronic hepatitis B. Aliment Pharmacol Ther 2015;43(4):458-69.

11. Wong GLH, Wong VWS, Choi PCL, Chan AWH, Chan HLY. Development of a non-invasive algorithm with transient elastography (Fibroscan) and serum test formula for advanced liver fibrosis in chronic hepatitis B. Aliment Pharmacol Ther. 2010;31(10):1095-103.

12. Eminler AT, Ayyildiz T, Irak K, Kiyici M, Gurel S, Dolar E, et al. AST/ALT ratio is not useful in predicting the degree of fibrosis in chronic viral hepatitis patients. Eur J Gastroenterol Hepatol. 2015;27(12):1361-66.

13. El-Hariri M, Abd El Megid AG, Taha Ali $\mathrm{TF}$, Hassany M. Diagnostic value of Transient Elastography (Fibroscan) in the evaluation of liver fibrosis in chronic viral hepatitis C: Comparison to liver biopsy. Egypt J Radiol Nucl Med. 2017;48(2):329-337.

14. Shiha G, Zalat K. Ishak versus METAVIR: Terminology, convertibility and correlation with laboratory changes in chronic hepatitis C. In: Liver Biopsy [Internet]. InTech; 2011.

15. Foucher J. Diagnosis of cirrhosis by transient elastography (FibroScan): a prospective study. Gut. 2006;55(3):403-8.

16. Degos F, Perez P, Roche B, Mahmoudi A, Asselineau J, Voitot $\mathrm{H}$, et al. Diagnostic accuracy of FibroScan and comparison to liver fibrosis biomarkers in chronic viral hepatitis: A multicenter prospective study (the FIBROSTIC study). J Hepatol. 2010;53(6):1013-21.

17. Oliveri F, Coco B, Ciccorossi P, Colombatto P, Romagnoli V, Cherubini B, et al. Liver stiffness in the hepatitis B virus carrier: A non-invasive marker of liver disease influenced by the pattern of transaminases. World J Gastroenterol. 2008;14(40):6154.

18. Chan HL-Y, Wong GL-H, Choi PC-L, Chan A W-H, Chim A M-L, You K K-L, et al. Alanine aminotransferase-based algorithms of liver stiffness measurement by transient elastography (Fibroscan) for liver fibrosis in chronic hepatitis B. J Viral Hep. 2009;16(1):36-44.

19. Marcellin P, Ziol M, Bedossa P, Douvin C, Poupon R, de Lédinghen V, et al. Non- invasive assessment of liver fibrosis by stiffness measurement in patients with chronic hepatitis B. Liver Int 2009;29(2):242-7.

20. Huang R, Jiang N, Yang R, Geng X, Lin J, $\mathrm{Xu} \mathrm{G}$, et al. Fibroscan improves the diagnosis sensitivity of liver fibrosis in patients with chronic hepatitis B. Exp Ther Med. 2016;11(5):1673-7.

21. Castera L. Hepatitis B: are non-invasive markers of liver fibrosis reliable? Liver Intl. 2013;34:91-6.

22. Seo YS, Kim MN, Kim SU, Kim SG, Um $\mathrm{SH}$, Han K-H, et al. Risk assessment of hepatocellular carcinoma using transient elastography vs. liver biopsy in chronic hepatitis B patients receiving antiviral therapy. Medicine. 2016;95(12):e2985.

23. Ansar M, Shafique U, Saeed F, Nadeem M, Khan MN. Evaluation of AST/ALT ratio as a marker of liver fibrosis and cirrhosis in patients with chronic hepatitis C. Pak Armed Forces Med J. 2010;59(4):429-432. Accessed November 23 , 2020 https://pafmj.org/index.php/PAFMJ/article /view/1745.

24. Williams ALB, Hoofnagle JH. Ratio of serum aspartate to alanine aminotransferase in chronic hepatitis Relationship to cirrhosis. Gastroenterology. 1988 Sep;95(3):734-9. 